\title{
Desenvolvimento clínico e estágio atual da odontologia adesiva
}

\author{
Bruna de Oliveira REIS ${ }^{1}$ \\ Glívia Queiroz LIMA ${ }^{\mathbf{1}}$ \\ Ana Teresa MALULY-PRONI ${ }^{2}$ \\ Henrico Badaoui Strazzi SAHYON ${ }^{2}$ \\ Thaís Yumi Umeda SUZUKI ${ }^{3}$ \\ Marco Aurélio de Lima VIDOTTI ${ }^{\mathbf{1}}$ \\ Erik Neiva Ribeiro de Carvalho REIS $^{4}$ \\ Eduardo Passos ROCHA ${ }^{2}$ \\ Wirley Gonçalves ASSUNÇÃO ${ }^{2}$ \\ Paulo Henrique DOS SANTOS ${ }^{2}$
}

Clinical development and present stage of adhesive dentistry

Desarrollo clínico y estágio actual de la odontología adhesiva

\begin{abstract}
${ }^{1}$ Departamento de Odontologia Restauradora, Faculdade de Odontologia, UNESP Univ. Estadual Paulista, 16105-050, Araçatuba-SP, Brasil ${ }^{2}$ Departamento de Materiais Odontológicos e Prótese, Faculdade de Odontologia, UNESP Univ. Estadual Paulista, 16105-050, Araçatuba - SP, Brasil

${ }^{3}$ Departamento de Odontologia Restauradora, Faculdade de Odontologia, Univ. Federal de Minas Gerais, 31270-901, Belo Horizonte - MG, Brasil

${ }^{4}$ Departamento de Cirurgia e Clínica Integrada, Faculdade de Odontologia, UNESP Univ. Estadual Paulista, 16105-050, Araçatuba-SP, Brasil
\end{abstract}

\section{Resumo}

Introdução: O maior foco das pesquisas odontológicas nos últimos 60 anos tem sido a adesão e suas técnicas. Mais de 7000 artigos já foram publicados a este respeito. O desenvolvimento dos materiais odontológicos adesivos e as técnicas a eles relacionadas possuem uma história interessante, onde descobertas do passado ainda são usadas de alguma forma no presente. Objetivo: expor, através de uma revisão de literatura, um breve histórico sobre materiais e técnicas restauradoras, bem como o estágio atual da odontologia adesiva, com ênfase na tradução de evidências baseadas em pesquisas laboratoriais para a prática clínica. Materiais e Métodos: Foram selecionados livros de preferência do autor para a introdução de conceitos clássicos e artigos de revisão publicados nos últimos 10 anos, utilizando as cinco palavras-chave: "Dental Bonding" AND "Dental Cements" AND "Resin Cements" AND "Adhesives" AND "Ceramics", sorteados pela melhor combinação na plataforma Pub/Med/MEDLINE. Resultados: Duzentos e um artigos, foram encontrados, sendo utilizados para análise qualitativa e quantitativa aqueles pertinentes ao direcionamento do autor, de acordo com o tema. Conclusão: Considerando as limitações do estudo, concluiu-se que a odontologia adesiva é uma área que segue em constante desenvolvimento, fundamental para a realização de restaurações minimamente invasivas e estéticas. Onde para que seja possível consequentemente longevidade clínica, os materiais utilizados e substrato dentário requerem conhecimento do profissional e fidelidade na execução de um correto pré-tratamento das superfícies, respeitando suas naturezas e composições.

Descritores: Colagem Dentária; Cimentos Dentários; Cimentos de Resina; Adesivos; Cerâmica.

\section{Abstract}

Introduction: The biggest goal of dental research in the last 60 years has been the adhesion and its techniques. More than 7,000 articles have already been published in this regard. The development of dental materials and related techniques are an interesting novel, where past discoveries are still used in the present. Objective: to expose, through a literature review, a brief history of materials and restorative techniques, as well as the current status of adhesive dentistry, with emphasis on translating evidence based on laboratory research into the clinical practice. Materials and Methods: It was selected books of the author's preference for the introduction of classic concepts and review articles published in the last 10 years using the five key words: "Dental Bonding" AND "Dental Cements" AND "Resin Cements" AND "Adhesives" AND " Ceramics ", sort by the best match on the Pub / Med / MEDLINE platform. Results: Two hundred and one articles were found, being used for qualitative and quantitative analysis those pertinent to the author's direction, according to the theme. Conclusion: Considering the limitations of the study, it was concluded that adhesive dentistry is an area that is constantly developing, fundamental for minimally invasive and aesthetic restorations. Where to clinical longevity be possible, the materials used and dental substrate require professional knowledge and fidelity in the execution of a correct pretreatment of the surfaces, respecting their natures and compositions.

Descriptors: Dental Bonding; Dental Cements; Resin Cements; Adhesives; Ceramics.

\section{Resumen}

Introducción: El mayor foco de las investigaciones dentales en los últimos 60 años ha sido la adhesión y sus técnicas. Más de 7000 artículos ya se han publicado a este respecto. El desarrollo de los materiales odontológicos adhesivos y las técnicas a ellos relacionados poseen una historia interesante, donde descubrimientos del pasado todavía se utilizan de alguna forma en el presente. Objetivo: exponer, a través de una revisión de literatura, un breve historial sobre materiales y técnicas restauradoras, así como la práctica actual de la odontología adhesiva, con énfasis en la traducción de evidencias basadas en investigaciones de laboratorio para la práctica clínica. Materiales y Métodos: Se seleccionaron libros de preferencia del autor para la introducción de conceptos clásicos y artículos de revisión publicados en los últimos 10 años, utilizando las cinco palabras clave: "Dental Bonding" AND "Dental Cements" AND "Resin Cements" AND " Adhesives "AND" Ceramics ", sorteados por la mejor combinación en la plataforma Pub/Med/MEDLINE. Resultados: Doscientos y un artículo, fueron encontrados, siendo utilizados para análisis cualitativo y cuantitativo aquellos pertinentes al direccionamiento del autor, de acuerdo con el tema. Conclusión: Considerar las limitaciones del estudio, se consideró que la adhesión de la demencia es un área que es necesaria para el desarrollo, fundamental para la invasión mínima y las renovaciones estéticamente. En el caso de la longevidad, es posible que los materiales utilizados y la dental dental requieren un buen conocimiento y fidelidad en la ejecución de la correcta pretreatment de la superficie, respecting sus naturas y composiciones.

Descriptores: Recubrimiento Dental Adhesivo; Cementos Dentales; Cementos de Resina; Adhesivos; Cerámica.

\section{INTRODUÇÃO}

Uma das condições fundamentais para o sucesso das restaurações na Odontologia é a adaptação adequada do material restaurador ao elemento dentário. Existem basicamente três mecanismos para que uma restauração seja fixada a uma cavidade: retenções microscópicas, retenções micro-mecânicas e ligações químicas, também conhecidas como "adesão verdadeira". As primeiras geralmente ocorrem nas restaurações de amálgama. As segundas podem ser encontradas nas restaurações de ionômero de vidro e resina composta, bem como procedimentos indiretos com utilização de cimentos 
resinosos, enquanto o terceiro tipo, supostamente, apenas nas restaurações à base de ionômero ${ }^{1,2}$.

Foi a partir do desenvolvimento da técnica de condicionamento ácido do esmalte sugerida por Buonocore em 1955 e o início do uso de adesivos dentinários $^{3,4}$. que se cogitou a possibilidade de realmente ocorrer adesão das restaurações ao dente. O ideal seria que a restauração se tornasse parte integrante do dente, ou seja, que o material se unisse à estrutura dental de forma completa e estável. Porém, apesar da evolução dos materiais e técnicas, esse ideal ainda não foi alcançado ${ }^{5,6}$.

Apesar de suas limitações, os adesivos dentinários desempenham função extremamente importante nas técnicas de restauração dental. Por serem mais fluidos do que as resinas compostas, os componentes dos adesivos dentinários penetram e se difundem com mais facilidade nas microrretenções formadas em esmalte e dentina pelo condicionamento ácido, proporcionando complementação da retenção mecânica e vedação marginal. Podem ser utilizados para substituir os vernizes cavitários, quando usados como proteção pulpar no forramento de restaurações não adesivas, em cavidades não profundas. Além de serem bastante eficientes nas cimentações de restaurações indiretas com cimentos resinosos ${ }^{7}$. Problemas clínicos, como reincidência de cárie, deslocamento e queda da restauração, danos à polpa, e sensibilidade pós-operatória, podem ser prevenidos através de uma boa união entre a restauração e o dente $^{8}$.

Graças à evolução da odontologia adesiva, estruturas dentais, que em outras épocas eram desgastadas para a confecção de núcleos metálicos fundidos e coroas totais metálicas, metalocerâmicas ou metaloplásticas, são hoje conservadas ao máximo. Isso só é possível devido às propriedades dos agentes cimentantes, bem como outros materiais restauradores $^{2}$.

Portanto, para que seja possível compreender a fundo a adesão, é necessário conhecer detalhadamente os tecidos dentários envolvidos no processo - esmalte e dentina - bem como possíveis materiais restauradores ${ }^{2}$, uma vez que o sucesso na odontologia adesiva dependerá destes conhecimentos, do conhecimento científico dos fenômenos da adesão, e, principalmente, de sua aplicação correta na prática clínica 9 .

O presente estudo tem como objetivo expor, através de uma revisão de literatura, um breve histórico sobre materiais e técnicas restauradoras, bem como o estágio atual da odontologia adesiva, com ênfase na tradução de evidências baseadas em pesquisas laboratoriais para a prática clínica.

MATERIAL E MÉTODO

- Procedimento e estratégia de busca

A revisão de literatura foi realizada por um autor, seguindo modelos propostos na literatura ${ }^{10,11}$. Foram selecionados 4 livros de preferência do autor para a introdução de conceitos clássicos. A seleção dos artigos foi direcionada aos artigos de revisão publicados nos últimos 10 anos, utilizando as cinco palavras-chave: "Dental Bonding" AND "Dental Cements" AND "Resin Cements" AND "Adhesives" AND "Ceramics", sorteados pela melhor combinação na plataforma Pub/Med/MEDLINE. Todos os resumos e textos completos foram obtidos através da mesma. A avaliação da qualidade metodológica foi baseada nos conceitos do PRISMA. ${ }^{10}$

\section{RESULTADOS E DISCUSSÃO}

Após a pesquisa na base de dados, de acordo com a metodologia, foram encontrados 201 artigos, sendo utilizados para análise qualitativa e quantitativa, aqueles pertinentes ao direcionamento do autor, de acordo com o tema.

- Desenvolvimento clínico e status atual da odontologia adesiva

\section{Substratos: esmalte e dentina}

O esmalte pode ser considerado uma estrutura homogênea, com aproximadamente $95 \%$ de conteúdo inorgânico (basicamente hidroxiapatita), $1 \%$ de conteúdo orgânico e $4 \%$ de água. É constituído por prismas paralelos entre si, e orientados de tal forma que chegam à superfície formando um ângulo de, aproximadamente $90^{\circ}$. Em algumas regiões dos dentes, os prismas não chegam à superfície que, neste caso, é formada por uma estrutura aprismática. ${ }^{5}$ Segundo Gwinnett $^{12}$, o esmalte aprismático pode ser encontrado em toda a extensão da superfície coronária dos dentes decíduos e é comumente encontrado em fóssulas, fissuras e regiões cervicais de dentes permanentes. É um esmalte desfavorável ao condicionamento ácido e, quando presente, deve ser desgastado para expor as extremidades dos prismas. Um pequeno desgaste superficial, como a confecção de um bisel nas margens dos preparos ou leve asperização da superfície, favorece muito a união do adesivo com o esmalte $^{13}$. Da mesma forma, para que haja retenção, as superfícies do esmalte devem estar livres de elementos externos, como: películas de origem embrionária (cutícula primária), películas adquiridas e placa bacteriana. Para tanto, o esmalte deve ser sempre limpo antes da realização de condicionamento ácido 5 . Além disso, em sistemas adesivos convencionais, na presença de esmalte aprismático, o ideal é que seja aumentado o tempo de condicionamento $^{2}$.

Outra característica estrutural do esmalte importante, é a presença das linhas incrementais de crescimento (originadas do repouso dos ameloblastos na deposição de matriz, e da mudança de orientação dos prismas), que estão relacionadas aos efeitos ópticos da luz ao incidir sobre o dente. Doenças 
típicas da infância também podem causar a interrupção da produção de matriz pelos ameloblastos, gerando alterações em sua mineralização, e consequentemente, pequenas manchas na estrutura do esmalte ${ }^{2}$.

Ao contrário do esmalte, a dentina pode ser considerada uma estrutura heterogênea com mais substâncias orgânicas (70\% de colágeno) e água (12\%) do que o esmalte, contendo apenas $18 \%$ de minerais (hidroxiapatita) ${ }^{5}$. Se caracteriza morfologicamente por uma rede de túbulos que se estendem desde a câmara pulpar até a junção amelodentinária. Quanto mais próximo à polpa, maior o número de túbulos encontrados ${ }^{14}$. $\mathrm{O}$ diâmetro dos túbulos também não é uniforme, é maior no limite dentino-pulpar (2,5 $\mathrm{mm})$ do que na periferia da dentina $(0,87 \mathrm{~mm})$. Consequentemente, a área ocupada pelos túbulos na junção amelodentinária é de apenas $1 \%$ da área total, enquanto, próximo da polpa, é de $22 \%{ }^{15}$. Além disso, a dentina apresenta variações estruturais, conforme a sua localização em relação à polpa, e modificações devidas a fatores externos, como a formação de dentina secundária, dentina de reparação, esclerose dos túbulos dentinários, etc. Essas modificações são provocadas por fatores como cárie, erosão, atrição, abrasão e, também, pela formação da camada compactada (smear layer) resultante do preparo do dente.

Além disso, pesquisas sobre enzimas intrínsecas da matriz dentinária, metaloproteinases (MMPs), foram realizadas e sua influência determinada ${ }^{16}$. As MMPs da dentina são ativadas durante o processo de lesão de cárie, levando à degradação de colágeno $^{17}$, e, por conseguinte, à progressão da lesão. Esta degradação do colágeno interfere no processo de remineralização da dentina, que é dependente de uma rede de colágeno adequada. Dentre os diversos papéis das MMPs estão: a participação na organização da matriz orgânica da pré-dentina e na regulação de sua mineralização, através do controle do volume de proteoglicanas; sua deposição no interior da dentina já mineralizada, podendo degradar o colágeno intratubular e outras proteínas $^{18}$; na progressão da cárie, podendo estar envolvidas na liberação e ativação de fatores de crescimento ligados à dentina, os quais ativam reações de defesa na polpa; durante a dentinogênese reparadora, podendo ter um papel na degradação da matriz, contribuindo para a formação da dentina reparadora atubular; participação na degradação da interface restauração-dentina; e, por fim, a possível participação na regulação da matriz extracelular do tecido pulpar em condições fisiológicas e patológicas $^{19}$.

Diferença na concentração de MMPs específicas foi relatada para dentina esclerótica em comparação à dentina sadia ${ }^{20}$. Na dentina esclerótica, embora a presença da MMP-2 seja reduzida, as
MMPs 8 e 9 são aumentadas ${ }^{20}$. Os mecanismos e implicações para essa observação ainda não são claros e podem ajudar a elucidar o processo de remineralização e a busca por abordagens terapêuticas mais biológicas. Também foram relatadas algumas alterações estruturais e químicas na dentina afetada por cárie em comparação à dentina sadia, como a diminuição do conteúdo de fosfato e carbonato, e a modificação na estrutura do colágeno pelo processo carioso ${ }^{21}$, onde a presença de fibrilas intactas de colágeno e proteoglicanas se mostraram significativamente menor do que na dentina sadia, sugerindo participação ativa das $\mathrm{MMPs}^{22}$. No entanto, a literatura ainda é escassa sobre as propriedades bioquímicas da dentina esclerótica e afetada por cárie em relação ao potencial de degradação do colágeno, e da influência dos inibidores de proteases sobre estes tipos de dentina.

Esmalte e dentina são dispostos em camadas e interligados por uma faixa chamada juncão amelodentinária (JAD), que atua como uma ponte entre o esmalte duro (E $70 \mathrm{GPa}$ ) e a dentina mais macia (E $20 \mathrm{GPa}$ ), permitindo uma transição de módulo de Young suave entre as duas estruturas. A fim de substituir a função mecânica do dente sob a perspectiva restauradora, não é apenas importante estudar os tecidos individualmente, mas também seu comportamento em conjunto. Assim, as propriedades mecânicas e características microestruturais do esmalte e da dentina, são importantes para a compreensão da dissipação de tensões no dente, execução de preparos, e desenvolvimento de materiais restauradores biomiméticos ${ }^{23}$.

\section{Sistemas adesivos}

Pode-se dizer que os adesivos dentinários são formados basicamente por uma resina fluida à base de BIS-GMA que tem por função o preenchimento dos poros entre as fibrilas colágenas, o selamento dos túbulos dentinários abertos (constituindo os tags resinosos), e a formação da camada híbrida, aderindo à restauração de resina composta sobrejacente ${ }^{1}$.

O desenvolvimento dos sistemas adesivos tem como enfoque a superação das adversidades de adesão inerentes ao tecido dentinário, bem como a simplificação da técnica para uso clínico ${ }^{24}$. Existem diversas classificações sugeridas por autores para os diferentes tipos de sistemas adesivos ${ }^{25}$. Algumas são baseadas no tipo de tratamento aplicado à camada de smear (total etch ou convencionais, e autocondicionantes), outras na cronologia do desenvolvimento (classificação por gerações), no número de passos clínicos de aplicação (three-step, two-step, single-step), ou ainda no número de frascos que primer e bond se apresentam (múltiplos frascos, one bottle e all-in-one).

Em relação aos passos clínicos, o princípio de todos os sistemas adesivos consiste em: condicionamento ácido do tecido dentário (que dentre 
outras, criará irregularidades superficiais no substrato), modificação do tecido condicionado através do uso de uma solução de monômeros diluídos em um solvente orgânico (primer) que prepara o tecido para a próxima etapa, a aplicação de uma resina fluida (adesivo ou bond) que permeará as irregularidades criadas pelo ácido, conferindo retenção micromecânica à restauração (hibridização). Quando as três etapas são realizadas sequencialmente, considera-se o adesivo como sendo de três passos. Quando um ou dois passos são simplificados, temos, respectivamente, os adesivos de dois passos e passo único. Ainda, a classificação pelo número de frascos considera os sistemas de três passos como múltiplos frascos, dois passos como one botle, e um único passo, como all-in-one ${ }^{2}$.

Os sistemas adesivos de múltiplos frascos são conhecidos como convencionais e foram os primeiros a serem criados. A partir da consolidação das etapas de "primerização" e "hibridização", surgiram os sistemas one bottle. Estes sistemas apresentam uma seringa contendo o ácido fosfórico e um frasco primer e bond juntos. Mais recentemente, a fim de minimizar os problemas provenientes da dificuldade de manutenção de uma umidade dentinária ideal para a difusão do primer/bond, da profundidade de desmineralização, e penetração do bond, os fabricantes passaram a suprimir uma das etapas mais sensíveis tecnicamente, o condicionamento ácido. ${ }^{2}$ Para isso, agregaram esta etapa à de "primerização" através do aumento da quantidade de monômeros ácidos na formulação dos primers. Assim, o condicionamento e aplicação do primer são realizados simultaneamente, e o enxágue com água após o condicionamento é eliminado ${ }^{26}$ Surgiram então, os sistemas adesivos com primers autocondicionantes (self-etching primers systems). Existe no mercado atualmente, um sistema adesivo onde o primer além de condicionante, tem ação antimicrobiana. Uma vez que nesses sistemas não é realizada a remoção da smear layer, que é incorporada na camada híbrida ${ }^{26}$, essa ação inibe o desenvolvimento de bactérias presentes nessa camada, e bactérias provenientes do meio bucal que se infiltrariam via interface dente-restauração. Tal primer é conhecido como brometo de metacriloiloxidodecilpiridina, o MDPB ${ }^{27}$.

Por fim, buscando facilitar ainda mais os procedimentos adesivos, foram desenvolvidos sistemas de passo único (all-in-one, multi-mode, universal $)^{28}$. Eles podem se apresentar de duas formas basicamente: agregando todos os componentes em um único frasco, podendo gerar relativa instabilidade no produto; ou em dois frascos, com os componentes em compartimentos separados que são misturados com o aplicador no momento de sua utilização ${ }^{2}$. São sistemas que provocam menor sensibilidade pós-operatória, porém uma das desvantagens é a menor preservação de esmalte intacto em relação ao uso do ácido fosfórico separadamente (efeito dependente do $\mathrm{pH}$ do adesivo), podendo diminuir a eficácia no que diz respeito à adesão $0^{29-31}$. Ainda, segundo Szesz et $\mathrm{al}^{32}$, por não produzirem o mesmo padrão de retenção, podem resultar em maior incidência de descoloração marginal nas restaurações, comprometendo a estética. Mas as pesquisas continuam evoluindo e estudos recentes tem buscado o desenvolvimento de materiais com propriedades mecânicas e biológicas mais satisfatórias, como os chamados "bioadesivos", que estão em fase de desenvolvimento ${ }^{33}$.

- Materiais restauradores

\section{Materiais restauradores diretos}

Atualmente, se tratando de odontologia minimamente invasiva direta, têm-se as resinas compostas e os cimentos de ionômero de vidro como materiais com propriedades adesivas ${ }^{2}$.

As resinas compostas são formadas por três fases: partículas inorgânicas, matriz resinosa, e agente de ligação silano nas partículas para produzir ligação entre esta e a matriz. O principal fator contribuinte para a confecção de cavidades minimamente invasivas é a adesividade das resinas compostas, principalmente em relação ao esmalte circundante que não necessita ser removido mesmo que sem suporte dentinário ${ }^{34,35}$. Diversos estudos se dedicam a aperfeiçoar as propriedades dos sistemas adesivos dentais e resinas compostas, especialmente a contração de polimerização e eficiência da formação da camada híbrida no tecido dental subjacente que ainda são fatores críticos e dependentes do operador $^{36}$.

Assim como das resinas compostas, o conhecimento das propriedades do ionômero de vidro é muito importante para o entendimento de sua aplicabilidade, uma vez que também possui espaço clínico amplo na odontologia minimamente invasiva. São de composição semelhante às resinas compostas, porém capazes de liberar flúor para a estrutura dental e de se recarregar com esses íons presentes no meio bucal, o que lhe confere propriedade anticariogênica a longo prazo. Possuem capacidade de adesão tanto a tecidos mineralizados como em componentes orgânicos da dentina (fibras colágenas) através de trocas iônicas. Seu valor de coeficiente de expansão térmica linear é muito próximo ao da estrutura dental, contribuindo para a manutenção do vedamento marginal. Além de ser biocompatível ${ }^{2,35,37}$.

Em adição, em casos de restaurações de dentes fraturados utilizando o próprio fragmento de dente como material restaurador, estudo realizado por do Poubel et al. $^{38}$ afirma a necessidade de rehidratação do fragmento por 15 minutos antes da união (aplicando adesivo multimode) para a manutenção de umidade suficiente e para que haja aumento da resistência de união. 


\section{Materiais restauradores indiretos}

Para restaurações confeccionadas em meio extra-oral, existem atualmente dois sistemas básicos de uso indireto: as resinas compostas de uso laboratorial e as cerâmicas. Atualmente, os compósitos indiretos podem ser classificados de acordo com suas características mais importantes, como: 1) o tipo de processamento (artesanal $\mathrm{x}$ industrial - blocos CAD-CAM), 2) sua microestrutura (com partículas dispersas $\mathrm{x}$ rede cerâmica infiltrada por polímeros), 3) forma de polimerização (baixa temperatura, alta temperatura, ou alta temperatura e pressão), 4) composição da matriz resinosa, 5) o tamanho das partículas e volume de conteúdo ${ }^{39}$.

As resinas laboratoriais, ou cerômeros, têm sido utilizadas por mais de 20 anos na Odontologia. Surgiu para minimizar problemas inerentes das resinas compostas de uso direto, como a grande contração de polimerização e sensibilidade técnica ${ }^{40}$. São denominadas pelos fabricantes como polímeros de vidro, cerômeros, polividros, ou porcelanas de vidro poliméricas. Podem ser indicadas para a confecção de facetas laminadas, inlays, onlays, overlays, coroas metaloplásticas, próteses fixas com infra-estrutura metálica, próteses sobre implantes com infra-estrutura metálica, coroas posteriores e próteses fixas reforçadas com fibra. ${ }^{2}$ Dentre outras, a possibilidade de reparo intra-oral é uma vantagem do material. Segundo Bottino et al. ${ }^{5}$, algumas das desvantagens são a necessidade de restaurações provisórias e o custo semelhante ao de restaurações cerâmicas, quando a necessidade de uso de cimentos resinosos.

Originada da palavra grega Keramos, as cerâmicas mais antigas feitas pelo homem datam de 26 mil anos atrás. Eram constituídas de gordura e ossos de animais misturados com cinza de osso e um material fino similar à argila. Depois de formadas, as cerâmicas eram queimadas sob temperaturas entre 500 e $800^{\circ} \mathrm{C}$, em fornos parcialmente enterrados na terra com paredes de limolita. ${ }^{41,42}$. Acredita-se que a fabricação de vidro esteja intimamente ligada à fabricação de cerâmica, que floresceu no Alto Egito cerca de 8000 anos a.C. Durante a queima da cerâmica, a presença de areia contendo óxido de cálcio $(\mathrm{CaO})$ combinada com óxido de sódio $(\mathrm{Na} 2 \mathrm{O})$ e o superaquecimento do forno de cerâmica pode ter resultado em um glaze colorido sobre a superfície da cerâmica. Especialistas acreditam que não foi antes de 1500 a.C. que o vidro foi produzido separadamente da cerâmica e obtido em diferentes formas $^{42,43}$.

A cerâmica odontológica, também conhecida como porcelana dentária, é considerada atualmente a melhor escolha para se reproduzir dentes naturais. $\mathrm{O}$ interesse pelas cerâmicas como materiais de infraestrutura na odontologia baseia-se em seu baixo peso, propriedades ópticas notáveis, altos valores de dureza, inércia química e características tribológicas incomparáveis ${ }^{5,41,42,44}$. Em virtude da grande quantidade de cerâmicas disponíveis no mercado, o profissional deve conhecer a composição do material para que empregue-o com segurança.

Estruturalmente, as cerâmicas são divididas em três categorias principais, como segue: (1) cristalinas ou policristalinas (sem conteúdo vítreo), (2) vidros com partículas ou parcialmente cristalinos (contendo alto ou baixo teor de vidro), e (3) vidros amorfos. A maioria das cerâmicas cristalinas, exceto por cristal único, são realmente policristalinas porque são constituídas por um grande número de pequenos cristais, ou grãos, separados entre si por seus limites ou contornos. Os átomos estão ligados de maneira menos regular ao longo do limite do grão e, consequentemente, existe uma energia interfacial, ou energia limítrofe, semelhante à energia de superfície. Portanto, a região limítrofe dos grãos é mais reativa quimicamente do que os próprios grãos, e esse conceito tem sido utilizado para acentuar a observação de diferentes fases dos materiais, condicionando ligeiramente a superfície da cerâmica antes da análise microscópica. Este raciocínio também é aplicado na criação de superfícies cerâmicas com retenção micromecânica por meio de condicionamento ácido, melhorando a união às resinas ${ }^{42,45}$

Considerando a reatividade química aos ácidos, as cerâmicas podem ser tanto ácidoresistentes quanto ácido-sensíveis, de acordo com o grau de degradação superficial produzida pelos ácidos. As cerâmicas ácidos-sensíveis (ex., cerâmicas a base de feldspato, leucita e dissilicato de lítio) são condicionadas originando superfícies micromecanicamente retentivas. As cerâmicas ácidoresistentes (ex., os sistemas cerâmicos de alumina e zircônia infiltrados por vidro, cerâmicas de alumina densamente sinterizadas, e cerâmicas de zircônia tetragonal estabilizada com ítria - Y-TZP) não mostram degradação superficial ao condicionamento, impedindo uma união micromecânica confiável à resina ${ }^{5}$.

A estabilidade química, alta resistência à compressão, estética excelente e duradoura, e a biocompatibilidade com os menores índices de aderência de placa, são algumas das características incomparáveis das cerâmicas odontológicas. Além disso, o módulo de elasticidade $(\mathrm{E})$, a dureza $(\mathrm{H})$, a densidade $(\rho)$, a condutividade térmica $(k)$ e o coeficiente de expansão térmica $(\alpha)$ são propriedades de valores muito semelhantes para o esmalte dentário e a porcelana feldspática, que são frequentemente, os materiais antagonistas as restaurações cerâmicas. Esse comportamento semelhante entre as cerâmicas odontológicas e os tecidos dentários beneficia muito o prognóstico das restaurações ${ }^{2,41}$. Entretanto, a 
segurança biológica das cerâmicas é um tema muito estudado atualmente. Tal propriedade pode ser diferente mesmo dentro da mesma classe de materiais. Alguns estudos in vitro ${ }^{46,47}$ observaram que as cerâmicas de dissilicato de lítio apresentam maior citotoxicidade do que as demais ligas e compósitos, e que se tornam ainda mais citotóxicas após o polimento.

Do ponto de vista da engenharia biomédica, a evolução da cerâmica dental nos últimos 30 anos tem sido mais interessante. A introdução da cerâmica de zircônia abriu uma ampla gama de aplicações. O consenso, entretanto, está no cuidado em selecionar a mais alta qualidade do produto e rigoroso respeito das recomendações dos fabricantes, com atenção especial na temperatura de sinterização (processo pelo qual se produz as cerâmicas). O controle preciso da cinética de sinterização está se tornando interesse no desenvolvimento de nano-cerâmicas e compósitos cerâmicos, onde "janelas cinéticas" podem ser muito estreitas para a retenção de microestruturas de grão fino e prevenção de reações secundárias indesejadas $^{48}$. Registaram-se progressos notáveis neste processamento (ex.: técnicas CAD-CAM, impressão 3D, tratamento de superfície com plasma, etc.) e desenvolvimento ao longo dos últimos anos. É provável que novas descobertas ocorram em um futuro próximo ${ }^{49}$.

\section{- Princípios da adesão}

Podemos definir adesão como uma atração molecular (ou atômica) entre duas superfícies (substratos) em contato, promovida pela força de atração interfacial de diferentes moléculas (ou átomos). A adesão é diferente da coesão, que é a atração entre moléculas semelhantes dentro de uma substância. Os conceitos de adesão e coesão também são importantes para a distinção de falhas adesivas e coesivas. A adesão pode ser de natureza mecânica, física e química, ou ainda, uma combinação entre elas $^{50}$. Mecânica, quando um adesivo líquido infiltrase nas irregularidades de uma superfície, solidificando-se posteriormente. Física, quando a união se dá por atração de forças moleculares fortes entre duas substâncias de composições diferentes. Química, quando há uma reação intermolecular entre duas substâncias de composições análogas ${ }^{6}$.

Sempre que um adesivo é utilizado para unir dois materiais, e se solidifica durante a união, o processo é denominado união adesiva. Dessa forma, os adesivos são substâncias que promovem a adesão entre dois substratos (aderentes). Além disso, uma substância intermediária pode ser utilizada para permitir a união entre o adesivo e o aderente, e esse material é conhecido como agente de união (coupling agent), por exemplo, os silanos. Alternativamente, os materiais utilizados para modificar as características da superfície do substrato, facilitando a adesão, são conhecidos como condicionadores (primers). São exemplos o ácido fosfórico para condicionar esmalte dentário e dentina, e o ácido hidrofluorídrico (HF) para as cerâmicas. Infelizmente, a distinção entre primers e coupling agents é raramente feita na literatura odontológica e os dois termos são utilizados de forma indiscriminada ${ }^{2}$.

Embora se suponha que possa haver adesão ao cálcio ou ao colágeno, não existe comprovação científica de que haja uma adesão verdadeira. A chamada "adesão" dos materiais ao dente é, na grande maioria dos casos, retenção micromecânica por infiltração de monômeros de adesivos nas irregularidades da superfície dentária. ${ }^{51,52}$. Nakabayashi e Pashley ${ }^{24}$ concluíram que união química, se é que ocorre, tem uma contribuição mínima para a adesão entre a resina e a dentina.

São fatores que favorecem a adesão:

- Superfícies de alta energia, tanto do adesivo como dos dentes (ex.: superficies livres de contaminação e ausência de smear layer $)^{50}$.

- Capacidade de umectação da dentina pelas resinas $^{50}$.

- Primers com monômeros hidrofílicos (ex.: HEMA), que facilitam a penetração dos adesivos em superficie dentinária úmida.

De grande importância para uma boa união entre o adesivo e o dente é a capacidade de umectação do adesivo, isto é, a possibilidade dele difundir-se na superfície do esmalte. A capacidade de umectação é medida pela tensão superficial de um líquido em contato com uma superfície sólida. Essa propriedade pode ser aferida pelo ângulo de contato que o líquido forma com a superfície. Quanto menor o ângulo de contato, mais a superfície ficará molhada e vice-versa ${ }^{5}$. A capacidade de umectação é também função da energia superficial de um sólido - quanto maior essa energia, melhor a umectação. Por exemplo, a energia superficial do esmalte após o condicionamento por ácido fosfórico é mais do que o dobro da energia superficial do esmalte em condições normais $^{53}$. O esmalte condicionado é facilmente molhado pelos monômeros usados em restaurações de resina ${ }^{52}$. No entanto, a energia superficial pode ser prejudicada por contaminação de saliva e impurezas, como óleo das turbinas de alta velocidade e jatos de ar e água da seringa tríplice, prejudicando a adesão.

\section{Adesão em esmalte}

De todos os procedimentos que visam à união entre os materiais restauradores às estruturas dentais, o mais importante é, inegavelmente, o condicionamento ácido do esmalte ${ }^{54-56}$. Técnica desenvolvida por Buonocore ${ }^{57}$ em 1955, tratando o esmalte com ácido fosfórico a $85 \%$ por 2 minutos, demonstrou clinicamente a adesão de pequenas porções de resina acrílica em dentes de pacientes, verificando a permanência da resina in situ. É surpreendente observar que, por meio de um trabalho de pesquisa simples, sem aparelhagem sofisticada, ou 
análise estatística, Buonocore ${ }^{57}$ promoveu uma verdadeira revolução em vários procedimentos clínicos. Hoje, o condicionamento ácido é obtido através de soluções de ácido fosfórico mais diluídas $(35 \% \text { a } 37 \%)^{58}$ e tempos mais curtos (15 a 30 segundos) ${ }^{59}$.

$\mathrm{Na}$ técnica de condicionamento, o ácido, ao entrar em contato com o esmalte, dissolve seletivamente os cristais de hidroxiapatita, com áreas mais condicionadas do que outras. Isso se deve à orientação dos cristais dos prismas, ou seja, alguns cristais são mais atingidos do que outros conforme sua orientação em relação à superfície dos prismas. Além disso, o condicionamento ácido proporciona aumento da energia de superfície ${ }^{5}$.

O ácido pode desmineralizar tanto a região central dos prismas como a sua periferia, criando microespaços na superfície do esmalte. Ao aplicar uma resina de consistência fluida no esmalte condicionado, ela preenche esses microespaços ficando, após sua polimerização, mecanicamente retida no esmalte. A resina pode não só encapsular os cristais de esmalte, como também penetrar no interior dos mesmos, formando um verdadeiro compósito em que eles constituem, o preenchimento e a resina, a matriz sintética $^{23,60}$.

\section{Adesão em dentina}

Com o sucesso do condicionamento ácido do esmalte, que não só proporcionou retenção para as resinas, como também reduziu consideravelmente a infiltração marginal $^{61}$, seguiu-se, na década de 1960 , a busca por um adesivo para a dentina. A primeira tentativa foi usar o condicionamento ácido. Verificou-se então que as resinas penetravam profundamente na dentina formando raízes dentro dos túbulos dentinários, mas não proporcionavam retenção ${ }^{4}$. Isso se explica porque a dentina normal é bastante úmida e as resinas adesivas então utilizadas eram hidrofóbicas, não penetrando nas retenções criadas pelo ácido. Os adesivos mais recentes usam resinas hidrofílicas em sua composição, como o HEMA (hidroxi-etil-metacrilato), que são compatíveis com a dentina úmida.

Vários autores demonstraram que a adesão é menor na dentina mais profunda do que na dentina superficial $^{62-64}$. Pode-se concluir que, quanto mais próxima da polpa estiver a dentina, mais úmida ela será. Outro fator importante a considerar, é o plano de corte da dentina no preparo cavitário. Quando a dentina é cortada longitudinalmente, os túbulos dentinários ficam em "meia-cana" e, portanto, menos retentivos do que os cortados transversalmente. Assim, não devemos e esperar uma retenção e uma vedação uniformes em toda interface dente-material restaurador.

Os diferentes tipos de dentina, a maior quantidade de água em sua composição e a presença de fluido intertubular e smear layer, interferem no comportamento dos adesivos e devem ser considerados com atenção quando do seu uso. Sabese que a presença dessa umidade é prejudicial durante e após o procedimento adesivo. Entretanto, para sua aplicação odontológica, o adesivo deve ser capaz de se aderir a superfícies que podem não permitir total secagem e, mais importante, manter essa adesão, mesmo em contato contínuo com a umidade ${ }^{2}$. Portanto, a estrutura dentinária é o tecido que oferece maiores dificuldades para se obter adesão ${ }^{5}$.

\section{Adesão em cerâmica}

A princípio, poderíamos pensar que as "frágeis" restaurações de cerâmica não suportariam o trabalho no meio intra-bucal. Isso seria verdade se a restauração não estivesse unida à estrutura dentária, ou aos materiais restauradores remanescentes (ex., compósitos e metais), funcionando como um sistema integrado onde os diversos tipos de estresses originados das cargas mastigatórias e hábitos parafuncionais (ex., bruxismo) são distribuídos através do sistema devido a uma adesão apropriada. Este raciocínio é tão verdadeiro que a norma ISO 6872 classificou as cerâmicas de acordo com o uso clínico a que se destinam e fez a distinção entre restaurações cimentadas de forma adesiva e não$\operatorname{adesiva}^{5,41}$.

O aumento do conteúdo cristalino (partículas de alumina e zircônia) nas cerâmicas odontológicas melhorou significativamente as propriedades mecânicas desses materiais, permitindo a utilização mais previsível de restaurações em cerâmica pura. No entanto, o aumento do conteúdo cristalino resultou em cerâmicas ácido-resistentes, que possuem um melhor desempenho estrutural que as cerâmicas ácido-sensíveis, porém não podem ser condicionadas a fim de promover uma adequada união à resina. Para superar esse problema, os sistemas de jateamento com sílica ou silicatização (ex.: Rocatec e Cojet, 3M ESPE) estão sendo utilizados para criar uma camada de sílica sobre a superfície de metais e cerâmicas por meio do impacto em alta velocidade das partículas de alumina modificadas por sílica, que podem penetrar até $15 \mu \mathrm{m}$ dentro do substrato cerâmico ou metálico ${ }^{5,65}$. Porém, estudo recente realizado por Chen et al. ${ }^{66}$ comprova a interferência que a água pode causar nesta união, onde foi observada a hidrólise da ligação coordenada entre MDP e zircônia como responsável pela deterioração da integridade da união entre uma Y-TZP condicionada por MDP e uma resina de metacrilato. $O$ que indica a necessidade de estudos mais aprofundados sobre o assunto para maior segurança na aplicação clínica.

O sucesso clínico dos procedimentos adesivos à base de resina em restaurações e reparos cerâmicos, depende da qualidade e durabilidade da união entre cerâmica e a resina. Esta qualidade, por sua vez, depende dos mecanismos de união controlados, em parte, pelo tratamento das 
superfícies, e em parte, pelos materiais selecionados para uso ${ }^{67}$. Em relação aos materiais ideais para proporcionamento de adesão adequada, deve-se salientar a importância do uso de fotopolimerizadores adequados (como os polywaves) para que ocorra a maior conversão de monômeros em polímeros possível $^{68}$.

Diferentes primers e adesivos, associados com procedimentos específicos nas técnicas de adesão, reduziram drasticamente a microinfiltração e suas consequências. Os cimentos de ionômeros de vidro (CIV) e os agentes resinosos convencionais de cimentação foram por muito tempo as principais escolhas para a união das restaurações cerâmicas à estrutura dental remanescente, onde o CIV convencional e o CIV modificado por resina (CIVMR) eram usados com frequência para cimentar cerâmicas ácido-resistentes devido à facilidade de realização da técnica ${ }^{69}$. Já em meados dos anos 2000, como uma alternativa às opções tradicionais de cimentação, foram introduzidos no mercado os cimentos resinosos autoadesivos. O que facilitou ainda mais o processo de cimentação, minimizando o tempo clínico, uma vez que não requerem os passos de condicionamento ácido e primerização. Outra grande vantagem foi a redução da sensibilidade pósoperatória $^{70}$. Entretanto, deve-se ressaltar que tais vantagens tornam-se irrelevantes quando o cimento resinoso não atinge bom desempenho em termos de selamento e união ${ }^{69}$. Estudos tem mostrado que a resistência de união de determinados cimentos resinosos autoadesivos ainda é menor do que a de cimentos resinosos tradicionais ${ }^{71-76}$, mas que por outro lado, o revestimento do resmanescente dental com resina poderia melhorar tanto a resistência de união dos cimentos resinosos convencionais quanto dos autoadesivos ${ }^{69}$.

Atualmente, pesquisas tem procurado a aplicação de nanotecnologia nos materiais odontológicos a fim de promover melhorias físicas, mecânicas e biológicas. Estudo realizado por Najeeb et al. ${ }^{37}$ nesta linhagem, implementou modificações nos CIV convencionais a fim de melhorar suas propriedades (que são menos favoráveis do que as dos CIVMR) através da incorporação de nanopartículas e nanocerâmicas bioativas. Os resultados obtidos foram promissores e essa incorporação de partículas não só aumentou as suas propriedades mecânicas, como promoveu uma maior liberação de flúor e bioatividade, porém mais estudos clínicos ainda são necessários. Dessa forma, os cimentos mais eficientes e mais utilizados para todos os tipos de restaurações cerâmicas ainda são os compósitos a base de resina, especialmente os sistemas que contêm monômeros de 10metacriloiloxidecil-dihidrogenio-fosfato (MDP), que são indicados para cimentar cerâmicas ácidoresistentes e apresentam melhores propriedades físico-químicas ${ }^{5,66,68,77}$. Inclusive, estudo in vitro recente avaliando discrepância marginal em coroas totais cerâmicas cimentadas com cimentos resinosos e CIVMR, apontou os cimentos resinosos como mais efetivos na redução da discrepância marginal. ${ }^{78}$ É importante saber que a técnica e o sistema adesivo utilizado para a hibridização dentinária poderão afetar a resistência de união imediata de determinados cimentos ${ }^{79}$.

O silano tem sido utilizado para melhorar a união entre adesivos orgânicos e as cerâmicas ou metais, em várias indústrias desde os anos 1940. A tecnologia de revestimento de partículas inorgânicas de carga pelos organosilanos melhorou a união à matriz resinosa, causando uma grande influência na fabricação de resinas compostas. Essa tecnologia também melhorou a adesão química de restaurações cerâmicas com a resina ${ }^{5,41}$. Os agentes de união à base de silano unem-se ao $\mathrm{Si}-\mathrm{OH}$ na superfície cerâmica por reações de condensação e as ligações duplas de metil-metacrilato permitem união ao adesivo resinoso. Enquanto existir Si-OH disponível na superfície cerâmica, uma adesão satisfatória deverá ser alcançada. Portanto, se o objetivo for obter uma fina camada de silano sobre qualquer superfície cerâmica, o protocolo de aplicação deve considerar: as diferentes microestruturas cerâmicas, os tipos de silano, e os mecanismos para reduzir a espessura da camada, como o aquecimento do material ${ }^{5,41}$. Em contrapartida, estudos recentes indicam que $o$ aumento da rugosidade da superfície (ex.: embricamento micromecânico) é mais importante do que o condicionamento químico com o silano na melhora das propriedades de união ${ }^{39,80}$.

A adesão entre as cerâmicas dentárias e os compósitos à base de resina é resultado da interação físico-química na interface entre ambas ${ }^{2,42}$. O processo para assegurar retenção adequada, vedação marginal e durabilidade de restaurações indiretas depende fortemente da cimentação efetiva. Portanto, uma consideração cuidadosa deve ser feita ao selecionar os materiais de acordo com sua aplicação.

CONCLUSÃO

A partir da presente revisão de literatura, considerando as limitações do estudo, concluiu-se que a odontologia adesiva é uma área em constante desenvolvimento, fundamental para a realização de restaurações minimamente invasivas e estéticas, possíveis graças à união de materiais restauradores, como resinas compostas e cerâmicas, à estruturas dentárias ou outros materiais através de agentes de união. Para que seja possível obter excelência na resistência dessa união e, consequentemente longevidade clínica, os materiais utilizados e substrato dentário requerem conhecimento do profissional e correto pré-tratamento das superfícies, de acordo com suas natureza e composição. 


\section{REFERÊNCIAS}

1. Van Meerbeek B, De Munck J, Yoshida Y, Inoue $\mathrm{S}$, Vargas M, Vijay P, et al. Buonocore memorial lecture. Adhesion to enamel and dentin: current status and future challenges. Oper Dent. 2003;28:215-35.

2. Miyashita E, Fonseca AS. Odontologia Estética: O estado da arte. São Paulo: Artes Médicas; 2004.

3. Hagger O. Swiss Patent 27894 British Patent 687299, 1951.

4. Buonocore MG, Willeman W, Brudevold F. A Report on a resin composition capable of bonding to human dentin surface. J Dent Res. 1956;35:846-51.

5. Bottino MA, Faria R, Valandro LF. Percepção: estética em próteses livres de metal em dentes naturais e implantes. São Paulo: Artes Médicas, 2009.

6. Larson TD. Using multiple bonding strategies. northwest dent. 2015;94:33-9.

7. Helvey GA. Adhesive dentistry: the development of immediate dentin sealing/selective etching bonding technique. Compend Contin Educ Dent. 2011;32:22,24-32,34-5.

8. Hashimoto M, Ohno H, Kaga M, Endo K, Sano H, Oguchi $H$. In vivo degradation of resin-dentin bonds in humans over 1 to 3 years. J Dent Res. 2000;79:1385-91.

9. Mante FK, Ozer F, Walter R, Atlas AM, Saleh N, Dietschi D, et al. The current state of adhesive dentistry: a guide for clinical practice. Compend Contin Educ Dent. 2013;34:2-8.

10. Moher D, Liberati A, Tetzlaff J, Altman DG, PRISMA Group. Preferred reporting items for systematic reviews and meta-analyses: the PRISMA statement. Open Med. 2009;3:e123-30.

11.Bento A. Como fazer uma revisão de literatura: Considerações teóricas e práticas. Revista JA (Associação Acadêmica da Universidade da Madeira). 2012;65:42-4.

12. Gwinnett AJ. Structure and composition of enamel. Oper Dent. 1992;5:10-7.

13. Mondelli J, Furuse AY, Francischone AC, Pereira MA. Excelência estética e funcional das resinas compostas em dentes posteriores. São Paulo: Artes Médicas; 2004.

14.Garberoglio R, Bränström M. Scanning electron microscopy investigation of human dentinal tubules. Arch Oral Biol. 1976;21:355-62.

15.Pashley DH. Dentin, a dynamic substrate - A review. Scanning Microscopy. 1989;1:161-74.

16. Mazzoni A, Mannello F, Tay FR, Tonti GA, Mazzotti G, Di Lenarda R et al. Zymographic analysis and characterization of MMP-2 and -9 forms in human sound dentin. J Dent Res. 2007;86:436-40.

17.Mankovskaia A, Lévesque CM, Prakki A. Catechin-incorporated dental copolymers inhibit growth of Streptococcus mutans. J Appl Oral Sci. 2013;21:203-7.

18. Sulkala M, Larmas M, Sorsa T, Salo T, Tjäderhane L. The localization of matrix metalloproteinase-20 (MMP-20, enamelysin) in mature human teeth. J Dent Res. 2002;81:603-7.

19.Tjaderhane L, Palosaari H, Wahlgren J, Larmas M, Sorsa T, Salo T. Human odontoblast culture method: the expression of collagen and matrix metalloproteinases (MMPs). Adv Dent Res. 2001;15:55-8.

20.Wang DY, Zhang L, Fan J, Li F, Ma KQ, Wang $\mathrm{P}$, et al. Matrix metalloproteinases in human sclerotic dentine of attrited molars. Arch Oral Biol. 2012;57:1307-12.

21.Wang Y, Spencer P, Walker MP. Chemicalprofileof adhesive/caries-affected dentin interfaces using Raman microspectroscopy. J Biomed Mat Res. 2007;81A:279-86.

22.Suppa P, Ruggeri A Jr, Tay FR, Prati C, Biasotto M, Falconi M, et al. Reduced antigenicity of type I collagen and proteoglycans in sclerotic dentin. J Dent Res. 2006;85:133-37.

23. Madfa AA, Yue XG. Dental protheses mimic the natural enamel behavior under functional loading: A review article. Jpn Dent Sci Rev. 2016;52:2-13.

24. Nakabayashi N, Pashley DH. Hybridization of Dental Hard Tissues. Tokio: Quintessence Publishing, 1998.

25.Van Meerbeek B, Vargas M, Inoue S, Yoshida Y, Peumans M, Lambrechts $P$, et al. Adhesives and cements to promote preservation dentistry. Oper Dent. 2001;6:119-44.

26.Pashley DH, Carvalho RM. Dentine permeability and dentine adhesion. J Dent. 1997;25:355-72.

27.Imazato S, Tarumi H, Ebi N, Ebisu S. Citotoxic effects of composite restorations employing selfetching primers or experimental antibacterial primers. J Dent. 2000;28:61-7.

28. Alex G. Universal adhesives: the next evolution in adhesive dentistry? Compend Contin Educ Dent. 2015;36:15-26.

29. Muñoz MA, Luque I, Hass V, Reis A, Loguercio $\mathrm{AD}$, Bombarda $\mathrm{NH}$. Immediate bonding properties of universal adhesives to dentine. Journal of Dentistry. 2013;41:404-11.

30.Pashley DH, Tay FR. Aggressiveness of contemporary self-etching adhesives Part II: Etching effects on unground enamel. Dental Mater. 2001;17:430-44.

31.Rosa WL, Piva E, Silva AF. Bond strength of universal adhesives: A systematic review and meta-analysis. J Dent. 2015;43:765-76.

32.Szesz A, Parreiras S, Reis A, Loguercio A. Selective enamel etching in cervical lesions for self-etch adhesives: A systematic review and meta-analysis. J Dent. 2016;53:1-11.

33.Kord FP, Lee BP. Recent approches in designing 
bioadhesive materials inspired by mussel adhesive protein. J Polym Sci A Polym Chem. 2017;55:9-33.

34.Peters MC, McLean ME. Minimally Invasive Operative Care I. Minimal Intervention and Concepts for Minimally Invasive Cavity Preparations. J Ad Dent. 2011;3:7-16.

35.Tyas MJ, Anusavice KJ, Frencken JE, Mount GJ. Minimal Intervention Dentistry - A review. Int Dent J. 2000;50:1-12.

36.Roulet JF, Wilson NHF, Fuzzi M. Advances in Operative Dentistry - Contemporary clinical Practice. Oxford: Quintessence Books, 2000.

37.Najeeb S, Khurshid Z, Zafar MS, Khan AS, Zohaib S, Martí JM, et al. Modifications in Glass Ionomer Cements: Nano-Sized Fillers and Bioactive Nanoceramics. Int $\mathrm{J}$ Mol Sci. 2016;17:pii:E1134.

38.Poubel DLN, Almeida JCF, Dias Ribeiro AP, Maia GB, Martinez JMG, Garcia FCP. Effect of dehydration and rehydration intervals on fracture resistance of reattached tooth fragments using multimode adhesive. Dent Traumatol. 2017;33:451-7.

39. Mainjot AK, Dupont NM, Oudkerk JC, Dewael TY, Sadoun MJ. From Artisanal to CAD-CAM Blocks: State of the Art of Indirect Composites. J Dent Res. 2016;95:487-95.

40.Lacy AM. A critical look at posterior composite restorations. J Am Dent Assoc. 1987;114:357-62.

41.Anusavice KJ. Phillips' Science of dental materials: 11th ed. Philadelphia: W.B, 2003.

42.Bella Dona A. Adesão às cerâmicas: evidências científicas para o uso clínico. São Paulo: Artes Médicas, 2009.

43.Fairhurst CW. Dental ceramics: the state of the Science. Adv Dent Res. 1992;6:78-81.

44.Kurdvk B. Giuseppangelo Fonzi: Industrial fabrication promoter of porcelain prosthetics. J History Dent. 1999;47:79-82.

45.Jones DW, Wilson HJ. Some properties of dental ceramics. J Oral Rehabil. 1975;2:379-96.

46. Messer RL, Lockwood PE, Wataha JC, Lewis JB, Norris S, Bouillaguet S. In vitro cytotoxicity of traditional versus contemporary dental ceramics. J Prosthet Dent. 2003;90:452-58.

47.Zarone F, Ferrari M, Mangano FG, Leone R, Sorrentino R. Digitally oriented materials: focus on lithium disilicate ceramics. Int $\mathrm{J}$ Dent. 2016:9840594.

48. Shen Z, Nygren M. Microstructural prototyping of ceramics by Kinect engineering: applications of spark plasma sintering. Chem Rec. 2005;5:17384.

49.Denry I, Kelly JR. Emerging ceramic-based materials for dentistry. J Dent Res. 2014;93: 1235-42.

50.Baier RE. Principles of adhesion. Oper Dent. 1992;5:1-9.
51.Erickson RL. Surface interactions of dentin adhesive materials. Oper Dent. 1992;5:81-94.

52.Ruyter, IE. The chemistry of adhesive agents. Oper Dent. 1992;5-11.

53.Jendresen MD, Glantz PO, Baier RE, Eick JD. Microtopography and clinical adhesiveness of an acid etched tooth surface. An vivo study. Acta Odontolol Scand. 1981;39:47-53.

54.Van Meerbeek B, Perdigão J, Lambrechts $P$, Vanherie G. The Clinical performance adhesives. J Dent Res. 1998;26:1-20.

55.De Munck J, Van Landuyt K, Peumans M, Poitevin A, Lambrechts P, Braem M, et al. A critical review of the durability of adhesion to tooth tissue: methods and results. J Dent Res. 2005;84:118-32.

56. Matei R, Popescu MR, Suciu M, Rauten AM. Clinical dental adhesive application: the influence on composite-enamel interface morphology. Rom J Morphol Embryol. 2014;55:863-68.

57.Buonocore MG. A simple method of increasing the adhesion of acrylic filling materials to enamel surfaces. J Dent Res. 1955;34:849-53.

58. Chow LC, Brown, WE. Phosphoric acid conditioning of teeth for pit and fissure sealants. J Dent Res. 1973;1517-25.

59.Bastos PA, Retief DH, Bradley EL, Denys FR. Effect of duration on the shear bond strength of a microfill composite resin to enamel. Am J Dent. 1988;1:151-57.

60.Gwinnett AJ. Acid etching for composite resins. Dent Clin North Amer. 1981;25:271-89.

61.Retief DH. Are adhesives techniques suficiente to prevent microleakage? Symposium of Dental Materials, Pulp Biology Group, IADR, The Netherlands. 1986.

62. Causton BE. Improved bonding of composite restorative to dentin. Br Dent J. 1984;156:93-5.

63. Mitchen JC, Gronas DG. Effects of time after extraction and depth of dentin on resin dentin adhesives. J Am Dent Ass. 1986;113:285-89.

64.Heymann HO, Bayne SC. Current concepts in dentin bonding: focusing in dentin adhesion factors. J Am Dent Ass. 1993;124:27-36.

65.Dbradović-Djuricić K, Medić V, Dodić S, Gavrilov D, Antonijević D, Zrilić M. Dilemmas in zirconia bonding: a review. Srp Arh Celok Lek. 2013;141:395-401.

66.Chen C, Chen Y, Lu Z, Qian M, Xie H, Tay FR. The effects of water on degradation of the zirconia-resin bond. J Dent. 2017; pii: S03005712, 17, 30088-X.

67. Naumova EA, Ernst S, Schaper K, Arnold WH, Piwowarczyk A. Adhesion of different resin cements to enamel and dentin. Dent Mater J. 2016;35:345-52. 
68. Novais VR, Rapouso LH, Miranda RR, Lopes CC, Simamoto PC Júnior, Soares CJ. Degree of conversion and bond strength of resin-cements to feldspathic ceramic using different curing modes. J Appl Oral Sci. 2017;25:61-8.

69. Giannini M, Takagaki T, Bacelar-Sá R, Vermelho PM, Ambrosano GMB, Sadr A et al. Influence of resin coating on bond strength of self-adhesive resin cements to dentin. Dent Mat J. 2015;34:8227.

70.Ferracane JL, Stansbury JW, Burke FJ. Selfadhesive resin cements - chemistry, properties and clinical considerations. J Oral Rehabil. 2011; 38:295-314.

71.De Munck J, Vargas M, Van Landuyt K, Hikita $\mathrm{K}$, Lambrechts P, Van Meerbeek B. Bonding o fan auto-adhesive luting material to enamel and dentin. Dent Mater. 2004;20:963-71.

72. Abo-Hamar SE, Hiller KA, Jung H, Federlin M, Friedl KH, Schmalz G. Bond strength of a new universal self-adhesive resin lutin cement to dentin and enamel. Clin Oral Invest. 2005;9:1617.

73. Aguiar TR, Di Francescantonio M, Ambrosano GM, Giannini M. Effect of curing mode on bod strength of self-adhesive resin luting cements to dentin. J Biomed Mater Res B Appl Biomater. 2010;93B:122-7.

74.Asmussen E, Peutzeldt A. Bonding of dual-curing resin cements to dentin. J Adhes Dent. 2006;8:299-304.

75.Cantoro A, Goracci C, Papacchini F, Mazzitelli C, Fadda GM, Ferrari M. Effect of pre-cure temperature on the bonding potential of self-etch and self-adhesive resin cements. Dent Mater. 2008;24:577-83.

76.76.Hitz T, Stawarczyk B, Fischer J, Hämmerle $\mathrm{CH}$, Sailer I. Are self-sdhesive resin cement a valid alternative to conventional resin cements? A laboratory study of the long-term bond strength. Dent Mater. 2012;28:1183-90.

77.Özcan M, Bernasconi M. Adhesion to zirconia used for dental restorations: a systematic review and meta-analysis. J Adhes Dent. 2015;17:7-26.

78. Ganapathy D, Sathyamoorthy A, Ranganathan H, Murthykumar K. Effect of resin bonded luting agents influencing marginal discrepancy in all ceramic complete veneer crowns. J Clin Diagn Res. 2016;10:ZC67-ZC70.

79.Lorenzoni E Silva F, Pamato S, Kuga MC, Só MV, Pereira JR. Bond strength of adhesive resin cement with different adhesive systems. J Clin Exp Dent. 2017;9:96-100.

80. Spitznagel FA, Horvath SD, Guess PC, Blatz MB. Resin bond to indirect composite and new ceramic/polymer materials: a review of the literature. J Esthet Restor Dent. 2014;26:382-93.

\section{CONFLITO DE INTERESSES}

Os autores declaram não haver conflitos de interesse.

AUTOR PARA CORRESPONDÊNCIA

Paulo Henrique dos Santos

paulo.santos@unesp.br

Submetido em 17/11/2018

Aceito em 12/03/2019 